\title{
Predicting daily distribution of solar irradiation by neural networks
}

\author{
S. Seme, G. Štumberger and J. Pihler \\ University of Maribor \\ Faculty of Electrical Engineering and Computer Science \\ Smetanova 17, 2000 Maribor (Slovenia) \\ Phone: +386 2220 7179, Fax number: +3862 252 5481, e-mail: Sebastijan.Seme@uni-mb.si
}

\begin{abstract}
This work focuses on prediction of solar irradiation during the day. In order to predict half hourly solar irradiation during the day an artificial neural network is applied. The artificial neural network was trained using error backpropagation learning rule. Meteorological data measured during three years were used to form learning patterns. The trained artificial neural network was tested with different patterns. Some of them were new while the others were used in the training procedure. The comparison of measured and by the artificial neural network predicted daily distribution of solar irradiation shows a very good agreement for the clear days.
\end{abstract}

\section{Key words}

Daily distribution of solar irradiation; Prediction; Artificial neural networks; Meteorological parameters, Clear day

\section{Introduction}

Solar irradiation is a very important data for engineers who can, based on this data, define the incline and necessary number of solar modules for required power of the solar power plant. Measurement of meteorological parameters such as temperature, global irradiance, relative air pressure etc. in Slovenia is performed in the scope of Environmental Agency of the Republic of Slovenia (ARSO) in 22 point. In the cases, when the measurements of solar irradiation cannot be performed, it can be predicted by different solar models. In this way a good estimation of solar potential can be obtained.

Artificial neural network is an appropriate tool for solving real problems in the cases where classical methods are insufficient. The meteorological parameters are important parameters for indicating the amount of available solar irradiation. In this work an artificial neural networks is applied to predict daily distribution of solar irradiation considering extraterrestrial irradiation and meteorological parameters.

There exist many articles where artificial neural network is applied to predict average daily solar irradiation [1]-[5]. Only a few of them, [6] and [7], are focused on solar irradiation in discrete, one hour long, time intervals during the day.
In this work five different parameters such as extraterrestrial solar irradiation on a horizontal surface, solar zenithal angle, day in the year, temperature, and relative air pressure, given in a half hour long time intervals, are used as input for artificial neural network, which is applied to predict daily distribution of solar irradiation. The results presented in the paper are given for the town Maribor in Slovenia. For training of the artificial neural network measured meteorological data for clear days between January $1^{\text {st }} 2005$ and March $31^{\text {st }}$ 2008 are used. They are provided by ARSO. The comparison of measured and predicted daily distribution of solar irradiation shows a good agreement for clear days. However, some more work is needed to get acceptable agreement between measured and predicted daily distribution of solar irradiation for cloudy days.

\section{Data collection}

For training the artificial neural network, data like extraterrestrial solar irradiation, solar angle, temperature, relative air pressure and global irradiance are required. The first two are obtained by numerically while the last three are measured data provided by ARSO.

\subsection{Calculation of extraterrestrial solar irradiation on a horizontal surface and solar angle}

The extraterrestrial solar irradiation on a horizontal surface $H$ was calculated by (1):

$$
H=\int_{t_{0}}^{t_{0}+\Delta t} I_{0} e \sin \alpha(t) d t
$$

where $I_{0}$ is the solar constant $\left(I_{0}=1367 \mathrm{~W} / \mathrm{m}^{2}\right), e$ is the eccentricity correction factor (2),

$$
e=1+0.034\left[\cos \left(\frac{2 \pi n}{365}\right)\right]
$$

$n$ is the number of the day starting from the first of January. The solar zenithal angle $\alpha$ is given by (3), 


$$
\sin \alpha=\sin L \sin \delta_{s}+\cos L \cos \delta_{s} \cos h_{s}
$$

where $L$ is the latitude of the site (for Maribor $\left.L=46^{0} 33^{\prime}\right), \delta_{\mathrm{s}}$ is the solar declination and $h_{\mathrm{s}}$ is the hour angle. The solar declination and hour angle are given by (4) and (5).

$$
\begin{aligned}
& \delta_{s}=23.45 \sin \left[\frac{360(284+n)}{365}\right] \\
& h_{s}=\cos ^{-1}\left(-\tan L \tan \delta_{s}\right)
\end{aligned}
$$

The extraterrestrial solar irradiation on a horizontal surface $H$ and solar zenithal angle $\alpha$ data were collected every half hour for every day in the year.

\subsection{Meteorological data}

Fig. 1 shows the location of Slovenia and position of the

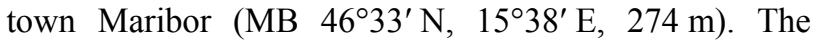
temperature, the relative air pressure and the global irradiance for town Maribor, measured by ARSO between January $1^{\text {st }} 2005$ and March $31^{\text {st }} 2008$ are shown in Figs 2, 3 and 4.

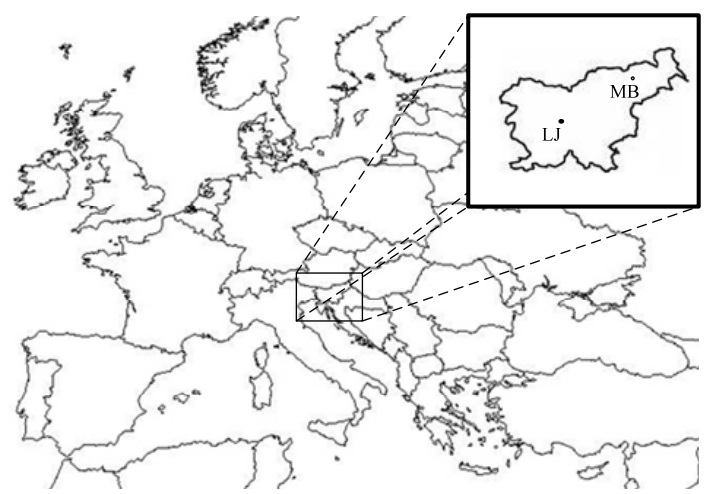

Fig. 1. Position of town Maribor (MB), Slovenia.

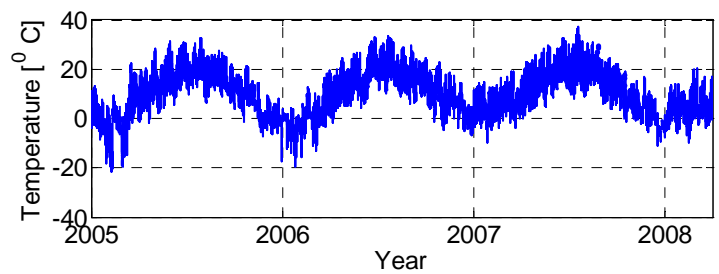

Fig. 2. Temperature in Maribor between January $1^{\text {st }} 2005$ and March $31^{\text {st }} 2008$.

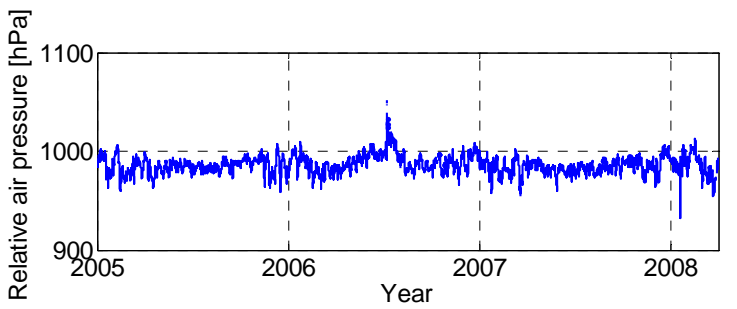

Fig. 3. Relative air pressure in Maribor between January $1^{\text {st }} 2005$ and March $31^{\text {st }} 2008$.

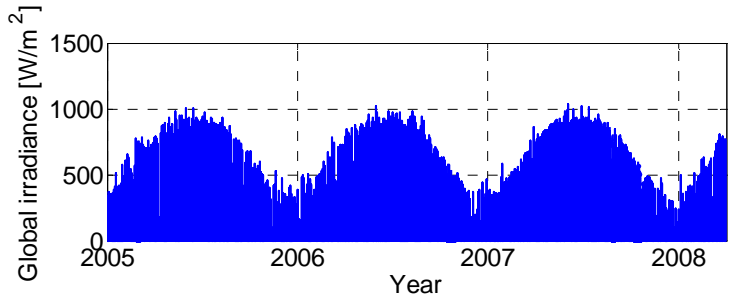

Fig. 4. Global irradiance in Maribor between January $1^{\text {st }}$ 2005 and March $31^{\text {st }} 2008$.

The data measured between January $1^{\text {st }} 2005$ and December $31^{\text {st }} 2007$ were used for training of artificial neural network, while data measured in year 2008 were used for testing the trained artificial neural network.

\section{Neural networks}

Neural networks are composed of simple elements operating in parallel. These elements are inspired by biological nervous systems. As in nature, the network function is determined largely by the connections between elements. Neural network can be trained to perform a particular function by adjusting the values of the connections (weights) between elements [8].

A neural network can contain several layers of neurons. Each layer has the weight matrix $\mathbf{W}$, the bias vector $\mathbf{b}$, and the output vector a. Fig. 5 shows how weights matrices, output vectors etc. are distinguished between themselves, for each of these layers. In Fig. $5 \mathbf{p}$ is the vector of $R$ inputs, $S$ is the number of neurons in each layer and $f$ is the transfer function. Non-linear sigmoid transfer functions $f$ were used. The weight matrices connected to inputs are called the input weights IW and weight matrices coming from layer outputs are called the layer weights $\mathbf{L W}$. The numbers of the layer are appended as a superscript to the variable of interest. For our case we use three-layer network shown in Fig 5 with 41 neurons (30 in the first layer, 10 in the second layer and 1 in the output layer).

The artificial neural networks accumulate knowledge during training process, while the effectiveness of artificial neural network depends on the quality of the training procedure. The goal of the training procedure is the minimal deviations between the target and calculated outputs. In this work the error backpropagation learning rule is applied and this rule consists of the following sequences [9]:

Step 1: Initialization of all weights and bias with small, randomly selected initial values.

Step 2: Calculation of vector output for all input training vector according to the equation (6) (Fig. 5.):

$$
\mathbf{a}=\mathbf{f}^{3}\left[\mathbf{L} \mathbf{W}^{3} \mathbf{f}^{2}\left[\mathbf{L} \mathbf{W}^{2} \mathbf{f}^{1}\left[\mathbf{I} \mathbf{W}^{1} \mathbf{p}^{+} \mathbf{b}^{1}\right]+\mathbf{b}^{2}\right]+\mathbf{b}^{3}\right]
$$




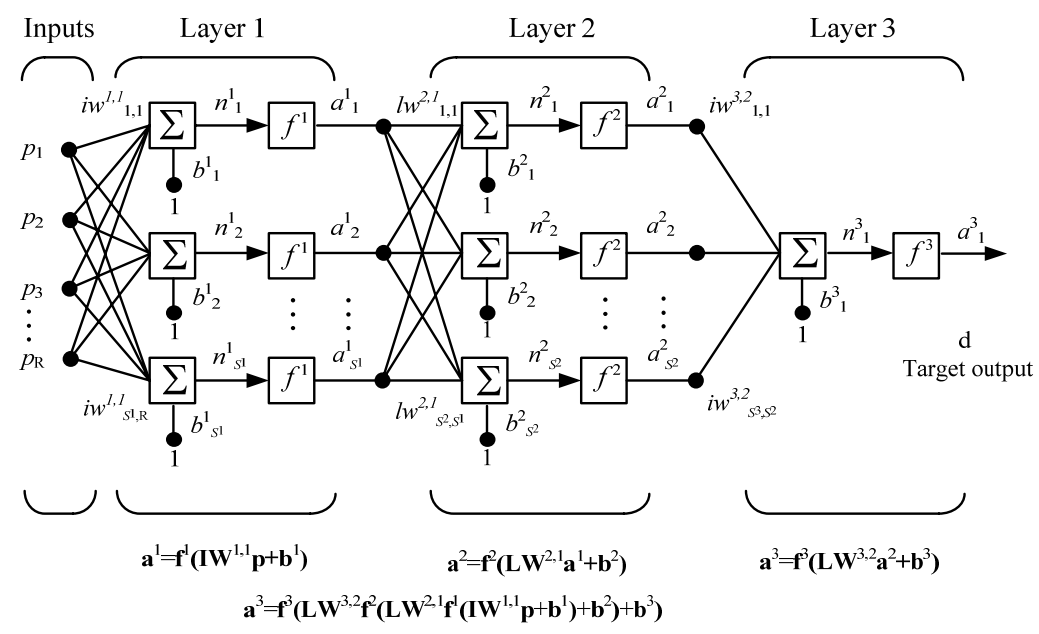

Fig. 5. Three layers artificial neural network.

Step 3: Calculation of the network error vector and the sum squared error for all the input vectors. Stop if the sum square error for all training vectors is less than the error goal, or if the specified maximum number of epochs has been reached, otherwise continue. Reverse calculation of partial errors with the help of previously determined target vector $\mathbf{d}$, and weight changes for all neuron layers.

Step 4: Calculation of each layer's new weight matrix and return to step 2 .

In this study calculation and meteorological indicators have been used for distribution of solar irradiation on horizontal surface. If more indicators are used, better results in output of the model can be achieved. In this work five input parameters and one target parameter were used to train the artificial neural network. The input parameters were the day in the year, the extraterrestrial solar irradiation on a horizontal surface, the solar angle, the temperature, and the relative air pressure, while the target parameter was measured global irradiance.

For the training, the data measured in 3 years, between January $1^{\text {st }} 2005$ and December $31^{\text {st }} 2007$, were used. From this period only clear days were selected. In the given case, the learning signal was composed of $74 \times 5$ patterns ( 74 clear days with 5 different inputs parameters Fig. 6). Figs. 7 and 8 show measured temperature and air pressure used in learning patterns while Fig. 9 shows target for artificial neural network measured solar global irradiation.

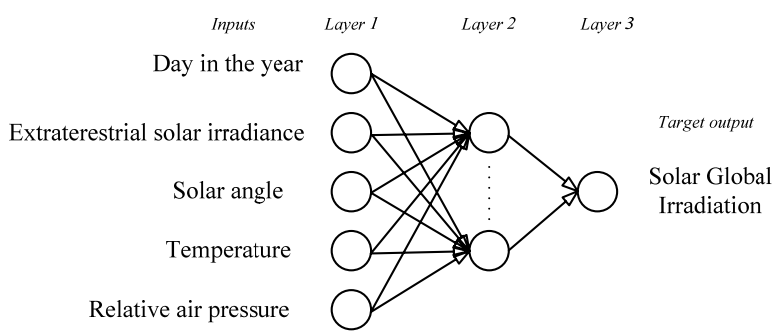

Fig. 6. Three-layer artificial neural network inputs and target.
For testing capability of the trained artificial neural network, data measured during 3 months in 2008 were used. From this period 10 clear days were selected in order to perform comparison between measured and by the artificial neural network predicted distribution of solar irradiation. The results are given in the next section.

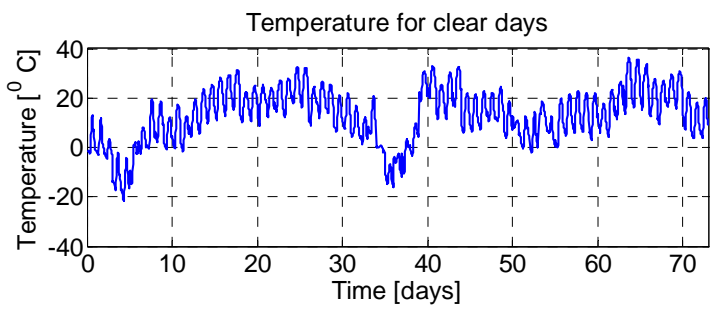

Fig. 7. Temperature for clear days between January $1^{\text {st }}$ 2005 and December $31^{\text {st }} 2007$.

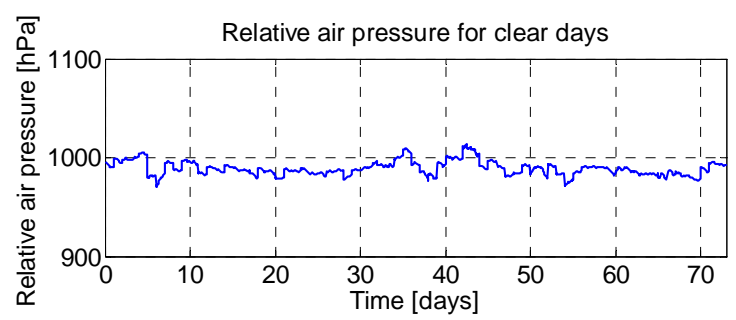

Fig. 8. Relative air pressure for clear between January $1^{\text {st }}$ 2005 and December $31^{\text {st }} 2007$.

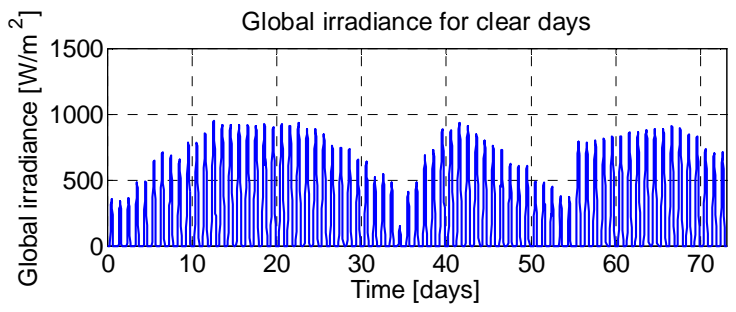

Fig. 9. Global irradiance for clear day between January $1^{\text {st }} 2005$ and December $31^{\text {st }} 2007$.

\section{Results}

Once the artificial neural network is trained, which means that all of weights and bias are set, it can be tested. 
First of all it can be tested with the patterns which were used in the training procedure. Fig. 10 shows measured and by the artificial neural network calculated global irradiance.

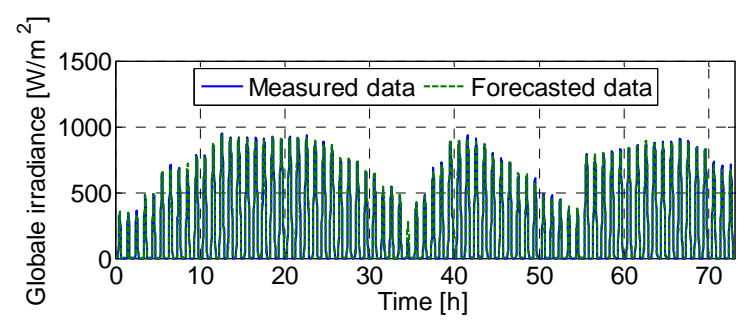

Fig. 10. Testing the trained artificial neural network with training patterns.

A comparison of measured and by the artificial neural network predicted global irradiance is presented point by point in Fig. 11. Better agreement of points presented in Fig. 11 with the straight line means also better agreement between measured and predicted points.

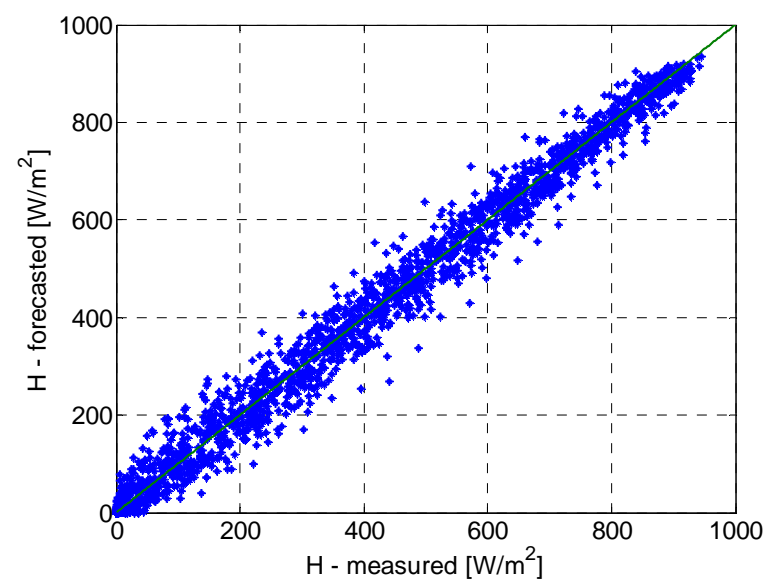

Fig. 11. Testing the trained artificial neural network with training patterns.

The comparison of the measured and predicted values shows that these values are aligned along the straight line. Quality of the trained artificial neural network can be evaluated only when it is tested also with the patterns that were not included in the training procedure. Fig. 12 shows comparison of measured and predicted global irradiance for the patterns that were not used in the training procedure. Different presentation of these results is shown in Fig.13.

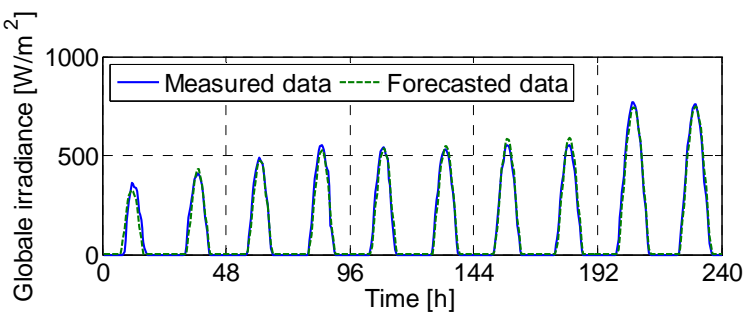

Fig. 12. Testing the trained artificial neural network with patterns which were not used in training.

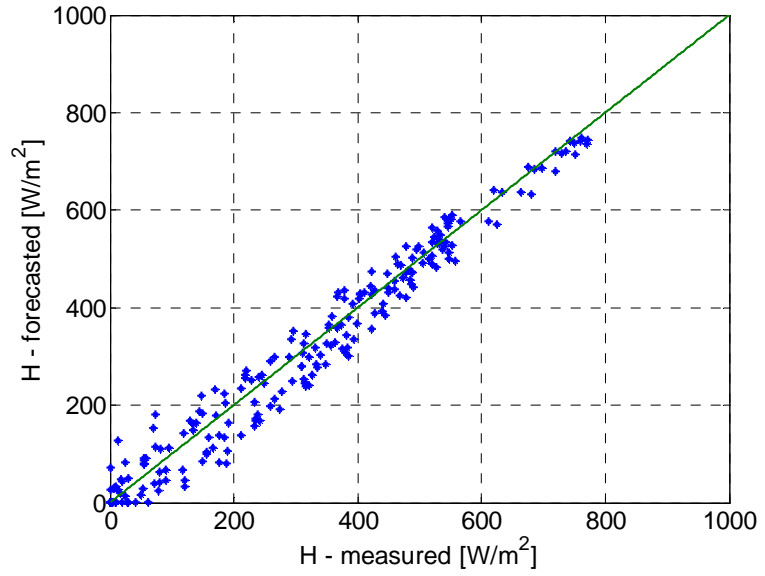

Fig. 13. Testing the trained artificial neural network with patterns which were not used in training.

The comparison of the measured and predicted values shows that the artificial neural network is well trained.

\section{Conclusions}

In this work daily distribution of global solar irradiance for clear days is predicted by the artificial neural network. The artificial neural network with 30 neurons in the first layer, 10 neurons in the second layer and 1 neuron in the third layer is used. It contains five inputs and one output. The inputs are calculated extraterrestrial solar irradiation, the day in the year, the solar angle, the temperature and the relative air pressure, while the output parameter is the global irradiance. For training of the artificial neural network measured data acquired in three years are used. Daily distribution of the global solar irradiance predicted by the trained artificial neural network is acceptable for clear days. However, some more work is needed to improve predicted daily distribution of global solar irradiance for cloudy days.

\section{References}

[1] J. L. De Souza, R. M. Nicacio, M. A. Moura, "Global Solar radiation measurements in Maceio, Brazil”, Renewable energy 30 (2005) 1203-1220.

[2] J. C. Cao, S. H. Cao, "Study of forecasting solar irradiance using neural networks with preprocessing sample data by wavelet analysis", Energy 31 (2006) 3435-3445.

[3] S. Pattanasethanon, C. Lertsatitthanakorn, S. Atthajariyakul, S. Soponronnarit, "An accuracy assessment of empirical sine model, a novel sine model and an artificial neural network model for forecasting illuminance/irradiance on horizontal plane of all sky types at Mahasarakham, Thailand", Energy Conversion and Management 49 (2008) 1999-2005.

[4] S. Cao, J. Cao, "Forecast of solar irradiance using recurrent neural networks combined with wavelet analysis", Applied Thermal Engineering 25 (2005) $161-172$ 
[5] A. Azadeh, A. Maghsoudi, S. Sohrab Khani, "Using an Integrated Artificial Neural Networks Model for Predicting Global Radiation: The Case Study of Iran", ICREPQ

[6] J. Cao, X. Lin, "Study of hourly and daily solar irradiation forecast using diagonal recurrent wavelet neural networks", Energy Conversion and Management 49 (2008) 1396-1406

[7] A. Sfetsos, A. H. Coonick, "Univariate and multivariate forecasting of hourly solar radiation with artificial intelligence techniques", Solar Energy Vol, 68, No. 2, pp. 169-178, 2000.

[8] Neural Network Toolbox, Matlab User's Guide, Version 5, 2006

[9] J. Pihler, B. Grčar, D. Dolinar, "Improved operation of power transformer protection using artificial neural network", IEEE Trans. On Power Delivery, Vol. 12, no. 3, pp. 1128-1136, 1997 